\title{
ORIGINAL ARTICLE \\ Contrasting the distribution of phenotypic and molecular variation in the freshwater snail Biomphalaria pfeifferi, the intermediate host of Schistosoma mansoni
}

\begin{abstract}
Y-NT Tian-Bi ${ }^{1,2}$, P Jarne ${ }^{3}$, J-NK Konan ${ }^{4}$, J Utzinger ${ }^{5,6}$ and EK N'Goran ${ }^{2,7}$
Population differentiation was investigated by confronting phenotypic and molecular variation in the highly selfing freshwater snail Biomphalaria pfeifferi, the intermediate host of Schistosoma mansoni. We sampled seven natural populations separated by a few kilometers, and characterized by different habitat regimes (permanent/temporary) and openness (open/closed). A genetic analysis based on five microsatellite markers confirms that $B$. pfeifferi is a selfer $(s \approx 0.9)$ and exhibits limited variation within populations. Most pairwise $F_{\mathrm{ST}}$ were significant indicating marked population structure, though no isolation by distance was detected. Families from the seven populations were monitored under laboratory conditions over two generations $\left(\mathrm{G}_{1}\right.$ and $\left.\mathrm{G}_{2}\right)$, allowing to record several life-history traits, including growth, fecundity and survival, over 25 weeks. Marked differences were detected among populations for traits expressed early in the life cycle (up to sexual maturity). Age and size at first reproduction had high heritability values, but such a trend was not found for early reproductive traits. In most populations, $\mathrm{G}_{1}$ snails matured later and at a larger size than $\mathrm{G}_{2}$ individuals. Individuals from permanent habitats matured at a smaller size and were more fecund than those from temporary habitats. The mean phenotypic differentiation over all populations $\left(Q_{S T}\right)$ was lower than the mean genetic differentiation $\left(F_{\mathrm{ST}}\right)$, suggesting stabilizing selection. However, no difference was detected between $Q_{\mathrm{ST}}$ and $F_{\mathrm{ST}}$ for both habitat regime and habitat openness.
\end{abstract}

Heredity (2013) 110, 466-474; doi:10.1038/hdy.2012.115; published online 16 January 2013

Keywords: Biomphalaria pfeifferi; population differentiation; life-history traits; microsatellite markers; $Q_{\mathrm{st}}-F_{\mathrm{st}}$ analysis

\section{INTRODUCTION}

Environmental structure lies at the heart of the adaptation process as the latter depends on both local (for example, genetic drift) and larger-scale (for example, migration) forces. This is especially true in spatially and/or temporally variable environment (Hartl and Clark, 1997; Bell, 2008). Analyzing the balance between genetic drift, mutation and migration in spatially structured populations based on neutral genetic markers has been the topic of tens of thousands of studies over the last decades (see Holsinger and Weir, 2009). However, understanding how adaptation proceeds, requires analyzing traits that are potentially under selection (for example, Bell, 2008). For example, populations living in different environments can evolve different lifehistory strategies or traits (Roff, 1997). The magnitude of selection in natural populations can be approached by complementing spatial studies of neutral variability with distribution patterns of traits. A popular approach has been to compare population structure at neutral markers versus selected traits, the so-called $F_{S T}$ versus $Q_{S T}$ approach (review in Merilä and Crnokrak, 2001; see Chapuis et al., 2007 for a recent reference). This approach may help unraveling the nature of selection. A basic prediction is that diversifying selection can be inferred when $F_{\mathrm{ST}}<Q_{\mathrm{ST}}$, whereas stabilizing selection is at play when $F_{S T}>Q_{S T}$. This approach has been criticized, especially with regard to the latter inference, because the respective values of $F_{\mathrm{ST}}$ and

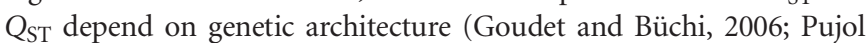
et al., 2008), relative genetic variability at neutral markers and traits (Edelaar et al., 2011), and for statistical reasons (Whitlock and Guillaume, 2009; Ovaskainen et al., 2011). It remains though useful as a first approach.

Other forces, both intrinsic and extrinsic to the organisms under focus, should be considered as well. An example of intrinsic force is the mating system, and we are here interested in the selfing rate. Selffertilization indeed strongly affects the action of drift/selection and migration, inducing variability loss and stronger population structure, compared to outcrossing situations (review in Charlesworth, 2003). Extrinsic forces include environmental variability, especially temporal variation in the availability of key resources. A fundamental empirical challenge is determining the extent to which the observed variation in life-history traits actually reflects adaptation to such variation (Keller and Douglas, 2008). An example of key resource for aquatic organisms is water availability, especially in freshwater environments, which might markedly vary in time (Dillon, 2000).

Freshwater snails of the Basommatophoran group offer excellent biological models to address these issues (Städler and Jarne, 1997; Charbonnel et al., 2002c; Chapuis et al., 2007; Escobar et al., 2008).

${ }^{1}$ Laboratoire de Génétique, UFR Biosciences, Université Félix Houphouët-Boigny, Abidjan, Côte d'Ivoire; ${ }^{2}$ Centre Suisse de Recherches Scientifiques en Côte d'Ivoire, Abidjan, Côte d'Ivoire; ${ }^{3}$ Centre d'Ecologie Fonctionnelle et Evolutive, UMR 5175, Montpellier, France; ${ }^{4}$ Centre National de Recherche Agronomique-Station LAME, Abidjan, Côte d'Ivoire; ${ }^{5}$ Department of Epidemiology and Public Health, Swiss Tropical and Public Health Institute, Basel, Switzerland; ${ }^{6}$ University of Basel, Basel, Switzerland and ${ }^{7}$ Laboratoire de Zoologie-Biologie Animale, Unité de Recherche et de Formation Parasitologie et Ecologie Parasitaire, UFR Biosciences, Université Félix Houphouët-Boigny, Abidjan, Côte d'Ivoire Correspondence: Dr Y-NT Tian-Bi, Laboratoire de Génétique, UFR Biosciences, Université Félix Houphouët-Boigny, 22 BP 1106 Abidjan 22 , Côte d'Ivoire.

E-mail: tianbyth@yahoo.fr

Received 20 November 2011; revised 11 October 2012; accepted 26 November 2012; published online 16 January 2013 
They occupy a variety of patchily distributed habitats along a gradient of hydrological permanence (Brown, 1994; Dillon, 2000). These hermaphroditic species reproduce through either self-fertilization or cross-fertilization (Escobar et al., 2011), offering the possibility of comparing contrasted mating systems with regard to population structure. Selfing species are less variable and more strongly structured than the outcrossing ones (Jarne, 1995; Escobar et al., 2011). Several studies using neutral genetic markers have also concluded that the population genetic structure at short spatial scale is affected by habitat characteristics (hydrological permanence or habitat openness; for example, Charbonnel et al., 2002c; Trouvé et al., 2003; Bousset et al., 2004). For example, less variation is maintained in closed (for example, ponds) than in open (for example, rivers) habitats (Bousset et al., 2004; Escobar et al., 2008). However, two studies only have compared neutral and phenotypic variation, the first in the lymnaeid Galba truncatula (Chapuis et al., 2007) and the second in the physid Physa acuta (Escobar et al., 2008). No study has untill now been conducted in the planorbids, the largest Basommatophoran family. Chapuis et al. (2007) showed that hydrological permanence may select for different optima in life-history traits $\left(Q_{\mathrm{ST}}>F_{\mathrm{ST}}\right.$ when contrasting permanent and temporary habitats). The results of Escobar et al. (2008) are also suggestive of diversifying selection on life-history traits in rivers and ponds.

We focus here on the tropical freshwater snail B. pfeifferi (Gastropoda, Pulmonata), the intermediate host of the parasitic trematode Schistosoma mansoni (the agent of human intestinal schistosomiasis) in Africa. This highly selfing species shows limited genetic variability within the populations (Charbonnel et al., 2002b,c, 2005 in Madagascar; Tian-Bi et al., 2008 in Côte d'Ivoire). Life-history traits have been monitored in a few studies under laboratory conditions (Loreau and Baluku, 1987; Tian-Bi et al., 2008), and little is known about their spatial variation in natural habitats. We gathered microsatellite data in seven populations separated by a few kilometers, and studied several traits over two generations under laboratory conditions using a family design, in order to characterize the spatial distribution of both neutral molecular markers and traits. We evaluated the genetic variance and heritability of traits, and compared the relative differentiation of markers and traits under different habitat characteristics (for example, water availability) for assessing how selection may affect the evolution of life-history traits in B. pfeifferi.

\section{MATERIALS AND METHODS}

\section{Species studied and sampling design}

B. pfeifferi is a freshwater snail distributed over most of Africa, Madagascar and the Middle East (Brown, 1994). This species occupies a variety of more or less permanent water bodies including streams, irrigation channels and dam lakes (Brown, 1994; Charbonnel et al., 2002a,b). Populations may experience density variation associated with flooding and drought, leading to bottlenecks and recolonization events (Jarne and Delay, 1991; Charbonnel et al., 2002a,b). Analysis of the genetic structure of natural populations (Charbonnel et al., 2002a,b,c), progeny-arrays analyses (Charbonnel et al., 2005) and laboratory experiments (Tian-Bi et al., 2008) showed that B. pfeifferi is a highly selfing species. A consequence of both population demography and the mating system is limited neutral variability within populations and fairly large genetic differentiation among populations (Charbonnel et al., 2002a). Individuals begin laying eggs when they are 2-3 months old (Loreau and Baluku, 1987; Tian-Bi et al., 2008). Isolated individuals readily lay eggs, and outcrossing is probably delayed compared to selfing (Tian-Bi et al., 2008). Egg capsules generally contain fewer than 10 eggs, and hatching occurs about 2 weeks after egg laying (Loreau and Baluku, 1987).

The study was carried out in the Man region (western Côte d'Ivoire). This area has been known to harbour populations of $S$. mansoni, the agent of intestinal schistosomiasis, for a few decades (Raso et al., 2005), suggesting recent invasion by B. pfeifferi. Seven sites were sampled in October 2004. Four sites (DOM, DOY, LYC and QTT) are located in the urban district of Man, whereas the remaining three sites (BLO, NIO and POD) are in nearby rural areas (Figure 1). Distances between sites range from a few hundred of meters to $23 \mathrm{~km}$. Their geographic coordinates are reported in Table 1 . Sites were classified according to hydrological characteristics (see Bousset et al., 2004) as temporary (dryed out in at least one visit during a one-year monthly survey) or permanent, and as closed (pond-like) or open (river-like). They were sampled using a long-handle sieve for a period of $10 \mathrm{~min}$, such as to collect at least 20 individuals per site. However, 7 and 11 individuals only were collected in the two sites, respectively, due to low density (Table 1). Snails collected in the field $\left(\mathrm{G}_{0}\right.$ generation) were brought alive to the laboratory.

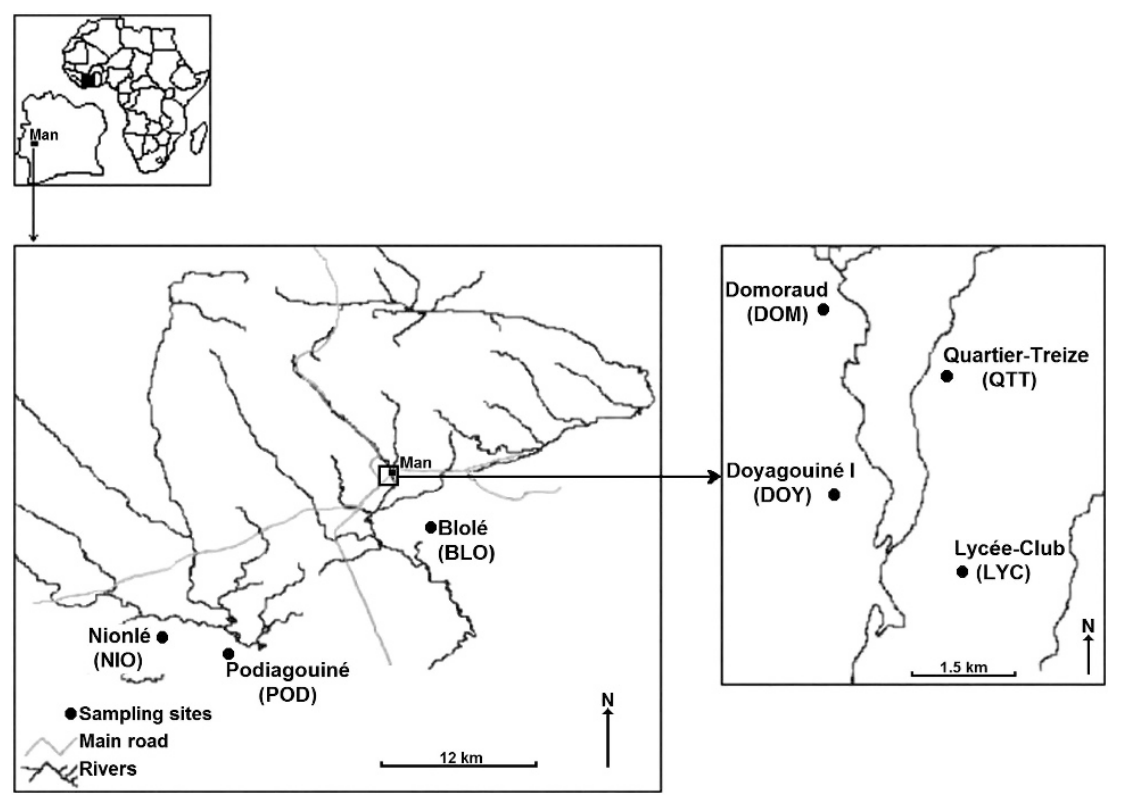

Figure 1 Location of the seven populations of $B$. pfeifferi sampled in the Man area, western Côte d'Ivoire. The populations sampled in the Man district are indicated in the enlarged panel. 
Table 1 Information on the seven studied populations of B. pfeifferi

\begin{tabular}{|c|c|c|c|c|c|}
\hline Population & Position & Type & $\begin{array}{c}\text { Habitat } \\
\text { openness }\end{array}$ & $\begin{array}{l}\text { Habitat } \\
\text { regime }\end{array}$ & $\mathrm{N}$ \\
\hline $\begin{array}{l}\text { Domoraud } \\
\text { (DOM) }\end{array}$ & $\begin{array}{l}07^{\circ} 24 / \\
07^{\circ} 33\end{array}$ & Irrigation canal & 0 & $\mathrm{~T}$ & 49 \\
\hline $\begin{array}{l}\text { Doyagouiné I } \\
\text { (DOY) }\end{array}$ & $\begin{array}{l}07^{\circ} 23 / \\
07^{\circ} 33\end{array}$ & $\begin{array}{l}\text { Rice field } \\
\text { (abandoned) }\end{array}$ & 0 & $\mathrm{~T}$ & 122 \\
\hline $\begin{array}{l}\text { Lycée-Club } \\
\text { (LYC) }\end{array}$ & $\begin{array}{l}07^{\circ} 23 / \\
07^{\circ} 32\end{array}$ & Irrigation canal & 0 & $P$ & 135 \\
\hline $\begin{array}{l}\text { Quartier-Treize } \\
\text { (QTT) }\end{array}$ & $\begin{array}{l}07^{\circ} 24 / \\
07^{\circ} 32\end{array}$ & Pond & C & $\mathrm{P}$ & 83 \\
\hline Blolé (BLO) & $\begin{array}{l}07^{\circ} 18 / \\
07^{\circ} 30\end{array}$ & River & 0 & $P$ & 21 \\
\hline Nionlé (NIO) & $\begin{array}{l}07^{\circ} 09 / \\
07^{\circ} 47\end{array}$ & Rice field & C & $\mathrm{T}$ & 11 \\
\hline $\begin{array}{l}\text { Podiagouiné } \\
\text { (POD) }\end{array}$ & $\begin{array}{l}07^{\circ} 09 / \\
07^{\circ} 44\end{array}$ & Fish pond & C & $P$ & 7 \\
\hline
\end{tabular}

Position refers to latitude (first line) and longitude (second line). T, P, O and C stand for temporary, permanent, open and closed, respectively. $N$ is the number of collected individuals.

\section{Analysis of population genetic structure}

The genetic analysis was conducted on $\mathrm{G}_{0}$ individuals. Total genomic DNA was extracted from foot tissues with the QIAmp DNA Mini Kit using standard protocols (QIAGEN, Inc., Hombrechtikon, Switzerland). Snails were genotyped at five microsatellite loci $(B p f 2, B p f 3, B p f 10, B p f 11$ and $B p f 12)$. These loci were chosen for their polymorphism and unambiguous amplification patterns (see Charbonnel et al., 2000 for detail). PCR amplifications were performed following multiplex protocols using the QIAGEN Multiplex PCR kit (QIAGEN, Inc.). PCR products were run on $5 \%$ acrylamide gels, at $55 \mathrm{~W}$ for $2 \mathrm{~h}$, concomitantly with a molecular size marker (EUROLADDER M, Courtaboeuf, France). Gels were stained with silver nitrate and allele size was determined visually.

The genetic variation within populations was quantified using several standard parameters including the number of polymorphic locus $\left(N_{\mathrm{pl}}\right)$, the mean number of alleles per locus $\left(N_{\mathrm{m}}\right)$, the mean observed heterozygosity $\left(H_{\mathrm{O}}\right)$ and gene diversity $\left(H_{\mathrm{E}}\right)(\mathrm{Nei}, 1987)$. Allelic frequencies were estimated per locus and population. Genotypic disequilibria between locus pairs and departures from Hardy-Weinberg equilibrium at each locus were tested within each population using exact tests (Rousset, 2007). Departure from HardyWeinberg equilibrium per population overall loci was evaluated using Fisher's method. The unbiased estimator $\hat{f}$ of Wright's inbreeding coefficient $F_{\text {IS }}$ was calculated according to Weir and Cockerham (1984). The selfing rate was estimated per population using the relationship $\hat{s}=2 \hat{f} /(1+\hat{f})$ (Hartl and Clark, 1997). In populations that were polymorphic at more than a single locus, the selfing rate $(s)$ was estimated by the $\mathrm{G}_{2}$ method (David et al., 2007) using RMES (available at http://ftp.cefe.cnrs.fr/). Genetic differentiation was assessed by calculating the estimator $\hat{\theta}$ of $F_{\mathrm{ST}}$ (Weir and Cockerham, 1984) overall loci for each population pair. Global $F_{S T}$ estimate was calculated over all populations. Isolation by distance was tested using a Mantel-like test on the relationship between genetic distance estimated by $F_{\mathrm{ST}} /\left(1-F_{\mathrm{ST}}\right)$ and the logarithm of geographic distance. All calculations and tests were performed using GENEPOP version 4.0 (Rousset, 2007).

\section{Analysis of life-history traits}

A general outline of the protocol is given in Figure 2. Twenty $\mathrm{G}_{0}$ adult snails were chosen at random per population (all snails in populations with lower sampling size), and isolated in $150 \mathrm{~cm}^{3}$ transparent plastic boxes. Egg capsules were collected daily over 3-7 days. After hatching, juvenile $G_{1}$ were maintained with their parents for $\sim 2$ weeks. Three $G_{1} s$ were collected at random in each box ( 7 and 10 in the samples with 11 and $7 \mathrm{G}_{0}$ snails, respectively). Sixty $\mathrm{G}_{1} \mathrm{~s}$ per population were randomly allocated to three 2 -1-tanks ( 20 individuals per tank), and were reared for 4 weeks. Thirty individuals were chosen at random (that is, 10 per tank) in each population when they reached $2.5-3.5 \mathrm{~mm}$ in size (that is, at 42 days), that is well before sexual maturity. The 210 individuals were thereafter isolated imposing reproduction through self-fertilization only. Several life-history traits of $\mathrm{G}_{1}$ individuals were monitored, including survival, fecundity and growth, over 25 weeks. Size (shell diameter) was measured weekly to the nearest $0.01 \mathrm{~mm}$ using an electronic (Digimatic) calliper. This allowed estimating size at first reproduction, as well as growth. Fecundity (number of eggs, of egg capsules and of eggs per capsule) was monitored twice a week, and individual survival three times a week. Capsules laid during week 18 after isolation were maintained in the boxes in which they were laid in order to generate the $\mathrm{G}_{2}$ generation. Egg capsules were checked every 2 days over 2 weeks in order to estimate the hatching rate. One week after hatching, two $G_{2}$ individuals per $G_{1}$ parent were chosen at random, isolated and reared for 17 weeks (until all reached sexual maturity), in the same conditions as their parents. We estimated their age and size at first reproduction. Fecundity at first reproduction was defined as fecundity (number of eggs, of egg capsules and of eggs per capsule) over 7 days after first egg laying. Throughout the experiment, snails were maintained at $21-24{ }^{\circ} \mathrm{C}$ (boxes) and $23-24^{\circ} \mathrm{C}$ (tanks) under a $12: 12$ photoperiod in dechlorinated tap water. They were fed with fish manufactured food. Food and water were changed at least twice a week.

Data analysis. From five to eight $\mathrm{G}_{1}$ individuals died during the course of the experiment, but most mortality occurred before week 10 (Supplementary Figure S1), that is shortly after reproduction began. Mortality did not differ among populations (Fisher's exact test, $P=0.811$ ). These individuals were not considered in the statistical analyses. Life-history traits were compared among populations using the generalized linear model (GLM) framework (Crawley, 2005). In both $G_{1}$ and $G_{2}$ individuals, continuous variables (age and size at first reproduction, growth parameters) were analyzed using a Normal error, and categorical variables (numbers of eggs, egg capsules and eggs per capsule) using a Poisson error (log link). The hatching rate of $G_{2}$ individuals was compared among populations using a Binomial error. Size data were used to build individual growth estimators based on the von Bertalanffy model. This model is characterized by two parameters, initial growth $(K)$ and final size $\left(S_{\infty}\right)$, and has been shown to appropriately describe growth in indeterminate growers, such as freshwater snails (Tsitrone et al., 2003). GLMs were also used to test for the effects of population, habitat regime/openness, generation and their interactions on early reproduction parameters. Spearman rank-order correlation coefficient was estimated between life-history traits in both $G_{1}$ and $G_{2}$ individuals, as well as between $G_{1}$ and $G_{2}$ snails for early reproduction parameters.

Within each population, regressions of offspring on parent (standardized values) were used to estimate the heritability of those five traits monitored in both generations (age and size at first reproduction, number of eggs, egg capsules and eggs per capsule at first reproduction) (see for example, Falconer and Mackay, 1996). As snails reproduced by selfing, heritabilities were directly obtained from the values of regression coefficients. Differentiation on quantitative traits $\left(Q_{\mathrm{ST}}\right)$ among populations within habitats was estimated as $Q_{\text {PopHab }}=\sigma_{\text {pop }}^{2} /\left(\sigma_{\text {pop }}^{2}+\sigma_{\text {fam }}^{2}\right)$, where $\sigma_{\text {pop }}^{2}$ and $\sigma_{\text {fam }}^{2}$ are the variance components among populations and among families within populations respectively (see for example, Chapuis et al., 2007; Escobar et al., 2008). The overall $Q_{\mathrm{ST}}$ was estimated as advocated by Chapuis et al. (2007). This approach gives equal weight to the traits considered and is consistent with the estimation of $F_{\mathrm{ST}}$ over all loci (see below). A confidence interval was generated for $F_{\mathrm{ST}}$ (with Genetix) and for $Q_{\mathrm{ST}}$ (with R) using a bootstrapping procedure on loci and individual trait values, respectively, based on 1000 replicates. The $Q_{S T}$ analysis was also conducted to compare habitats. Q estimated as $Q_{\text {HabTotal }}=\sigma_{\text {hab }}^{2} /\left(\sigma_{\text {hab }}^{2}+\sigma_{\text {pop }}^{2}+\sigma_{\text {fam }}^{2}\right)$, where $\sigma_{\text {hab }}^{2}, \sigma_{\text {pop }}^{2}$ and $\sigma^{2}$ fam are the variance components between habitats, among populations within habitats and among families within populations, respectively. Values were considered significantly different when their $95 \%$ confidence intervals did not overlap. All analyses of life-history traits were performed using STATA version 9.0 and the software R for Windows. All experiments were performed in accordance with relevant guidelines and regulations.

\section{RESULTS}

Molecular variation within and among populations

The loci studied exhibited from two to four alleles (Supplementary Table S1). Three of the seven populations studied were polymorphic 


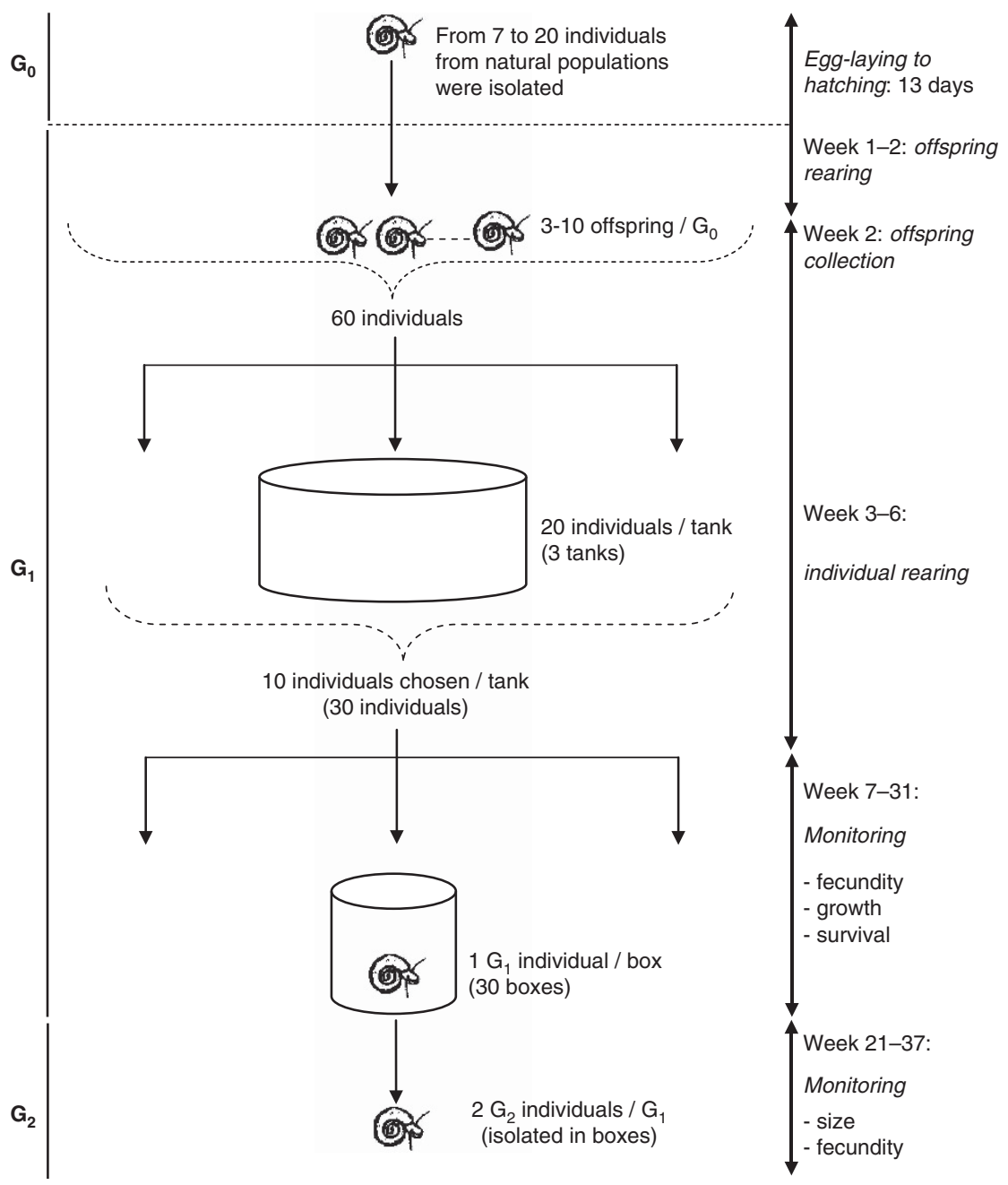

Figure 2 Schematic diagram of the experimental protocol conducted in the seven populations studied (more details in text). $G_{i}$ refers to generation. Seven to twenty field-caught $G_{0}$ individuals per population were isolated. Sixty $G_{1}$ offspring were chosen at random (from 3 to $10 G_{1}$ depending on sample size in natural populations), and set in three groups of 20 individuals on week 3 . At week 6,10 individuals were sampled per group (30 $\mathrm{G}_{1}$ individuals per population), and isolated for 25 weeks (week 7-31) during which fecundity, growth and survival were monitored. At week 18, egg capsules were collected to monitor the hatching rate of $G_{2}$ individuals. One week after hatching (10-13 days), two $G_{2}$ were randomly chosen per $G_{1}$ snail, and they were isolated in boxes for 17 weeks and monitored up to sexual maturity in order to quantify some life-history traits (size and fecundity).

at a single locus, and the four others had three to four polymorphic loci (Table 2). The mean number of alleles per locus and population ranged between 1.20 and 1.80 , and gene diversity from $0.02-0.24$. No heterozygotes were detected in three populations, and the highest value of observed heterozygosity was 0.07 in DOY (Table 2). The number of multi-locus genotypes was low, ranging from four to six in the four populations exhibiting polymorphism at more than a single locus. Genotypic disequilibria were detected for 15 locus pairs (out of 70 ), and the Bpf10-Bpf11 pair was involved twice in a significant test. Hardy-Weinberg equilibrium tests per locus and population were all significant $(P<0.05)$, as were all tests per population overall loci $(P<0.01)$. The estimates of $F_{\text {IS }}$ varied from 0.76 to 1 , producing selfing rates ranging between 0.86 and 1 . The selfing rate $\hat{S}$ based on RMES ranged between 0.90 and 0.96 (Table 2).

Differentiation tests over all populations and loci were highly significant $(P<0.001)$, and the corresponding global estimate of $F_{\mathrm{ST}}$ was 0.74 . The pairwise $F_{\mathrm{ST}}$ values over all loci ranged between 0.07 and 0.93 (Table 3 ). They all differed significantly from zero
$(P<0.001)$, except for that between DOY and QTT $(\hat{\theta}=0.07$, $P=0.062)$. No significant isolation by distance was detected $(r=0.26, P=0.274)$.

\section{Variation of life-history traits}

Significant differences in most traits associated to first reproduction in $G_{1}$ individuals were detected among populations (Table 4). For example, size at first reproduction ranged from $6.60 \mathrm{~mm}$ in QTT to $9.09 \mathrm{~mm}$ in DOM (note that there was no significant difference in size among populations when individuals were isolated; GLM, $P=0.415$ ), and age at first reproduction varied from 80.80 days in POD to 103.33 days in DOY. No significant difference was observed between fecundity parameters after the first reproduction (Table 4). The dynamics of egg laying was rather similar across the populations (Supplementary Figure S2), with a slow increase in the number of eggs laid up to a marked peak at 18-20 weeks of age (perhaps partly due to uncontrolled environmental variation), followed by presumably senescent decline. Although overall growth differed among 
Table 2 Population polymorphism at five microsatellite loci over the seven studied populations of $\boldsymbol{B}$. pfeifferi

\begin{tabular}{lcccccccc}
\hline Population & $\mathrm{N}$ & $\mathrm{N}_{p l}$ & $\mathrm{~N}_{m}$ & $\mathrm{H}_{O}$ & $\mathrm{H}_{E}$ & $\hat{f}$ & $\hat{\mathrm{s}}$ & $\hat{\mathrm{s}}^{\prime}$ \\
\hline DOM & 20 & 4 & $1.80(0.45)$ & $0.00(0.00)$ & $0.24(0.25)$ & 1.00 & 1.00 & 0.92 \\
DOY & 20 & 3 & $1.60(0.55)$ & $0.07(0.10)$ & $0.15(0.16)$ & 0.82 & 0.90 & 0.94 \\
LYC & 20 & 1 & $1.20(0.45)$ & $0.00(0.00)$ & $0.02(0.02)$ & 1.00 & 1.00 & - \\
QTT & 20 & 3 & $1.60(0.55)$ & $0.04(0.04)$ & $0.06(0.08)$ & 0.80 & 0.89 & 0.90 \\
BLO & 21 & 3 & $1.60(0.55)$ & $0.01(0.02)$ & $0.08(0.08)$ & 0.88 & 0.94 & 0.96 \\
NIO & 11 & 1 & $1.20(0.45)$ & $0.02(0.04)$ & $0.07(0.16)$ & 0.76 & 0.86 & - \\
POD & 7 & 1 & $1.20(0.45)$ & $0.00(0.00)$ & $0.05(0.12)$ & 1.00 & 1.00 & -
\end{tabular}

$N$ is the number of individuals analyzed. $N_{\mathrm{pl}}, N_{\mathrm{m}}, H_{0}, H_{\mathrm{E}}$ stand, respectively, for the number of polymorphic loci, the mean number of alleles, the mean observed heterozygosity and Nei's unbiased gene diversity over all loci. Their standard errors are indicated in parentheses. $f$ is the estimate of Wright's $F_{I S}$ and $\hat{s}$ the estimate of the selfing rate derived from $f . \hat{s}^{\prime}$ is the estimate of the selfing rate based on the method of David et al. (2007).

Table 3 Pairwise estimator of $F_{\mathrm{ST}}(\hat{\theta})$ among seven populations of B. pfeifferi (below the diagonal) and geographical distances (shortest distances) separating populations (in kilometer; above the diagonal)

\begin{tabular}{lccccccc}
\hline & DOM & DOY & LYC & QTT & BLO & NIO & POD \\
\hline DOM & & 0.82 & 1.81 & 1.13 & 7.59 & 22.39 & 20.58 \\
DOY & 0.58 & & 1.28 & 1.19 & 6.87 & 21.84 & 19.97 \\
LYC & 0.58 & 0.55 & & 1.10 & 5.84 & 22.32 & 20.32 \\
QTT & 0.70 & 0.07 & 0.82 & & 6.89 & 22.98 & 21.07 \\
BLO & 0.71 & 0.82 & 0.93 & 0.88 & & 21.36 & 18.87 \\
NIO & 0.56 & 0.55 & 0.90 & 0.69 & 0.81 & & 2.88 \\
POD & 0.53 & 0.55 & 0.91 & 0.75 & 0.87 & 0.61 & \\
\hline
\end{tabular}

populations, no difference was detected in the two parameters from the von Bertalanffy model. The variation in initial growth was marginally significant (Table 4), but final growth did not show significant variation despite values ranging from $7.9-12.5 \mathrm{~mm}$ (Supplementary Figure S3). No difference was detected among populations for the hatching rate of $\mathrm{G}_{2}$ individuals (Table 4). Of the five traits monitored in $G_{2}$ individuals, only the number of eggs showed significant differences among populations in both $G_{1}$ and $\mathrm{G}_{2}$ generations. A significant generation effect was detected for these five traits over all populations, traits in $G_{2}$ individuals showing lower values than those recorded in $G_{1}$ snails. Population-bygeneration interactions were also mostly (or nearly so) significant for these five traits, suggesting that there was no consistent trend in traits among populations. Correlations between traits are reported in Supplementary Table S2. On the whole, the few significant correlations essentially bear on reproduction (number of egg capsules, eggs and eggs per capsule), a classical result in freshwater snails that was also detected in $\mathrm{G}_{2}$ snails (Supplementary Table S3). Initial growth was negatively correlated with final size in all populations (Supplementary Table S2). No correlation was significant across generations after correction for multiple testing (Supplementary Table S4).

Habitat regime (permanent/temporary) affected some of the nine traits studied (Supplementary Table S5). Significant effects were detected for the three reproductive traits measured at first reproduction. Individuals from permanent habitats were, for example, smaller and produced more eggs at first reproduction than those from temporary habitats. A similar tendency was detected for reproduction later on in life (Supplementary Table S5). The effect on the number of eggs at the first reproduction was detected in $G_{1}$ individuals, though not in $\mathrm{G}_{2}$ (Supplementary Table S5). Differences were also detected with regard to habitat openness: individuals from open habitats reproduced later and at larger size, grew faster and reached a smaller final size that those from closed habitats (Supplementary Table S5). The number of eggs per capsule and number of eggs were also affected by generation. The marked effect in permanent and in open habitats (Supplementary Table S5) is due to low values in BLO, POD and QTT.

Age and size at the first reproduction showed significant heritability in most populations (Table 5). The other traits related to first reproduction differed significantly in a single population each. Four out of five traits showed significant heritability in the BLO population. $Q_{\mathrm{ST}}$ estimates per trait over all populations ranged between 0.35 and 0.68 producing a mean value of 0.52 (95\% CI: $0.40-0.61)$ (Table 6). This is less than the multi-locus $F_{\mathrm{ST}}$ values among populations $(0.74$; $95 \%$ CI: $0.65-0.80$; Table 6$)$. However, there was no significant difference between $Q_{\mathrm{ST}}$ and the corresponding $F_{\mathrm{ST}}$ for both habitat regime and habitat openness (overlapping 95\% CI; Table 6).

\section{DISCUSSION}

\section{Neutral variation in a selfer}

The genetic variability was extremely reduced, especially in two populations, confirming results in B. pfeifferi populations from Madagascar using the same markers (Charbonnel et al., 2002a,b). We also confirm (using both $F_{\text {IS }}$ and David et al.'s multi-locus method) that this species is a selfer with selfing rates $>90 \%$ (Charbonnel et al., 2005; Tian-Bi et al., 2008). Strong differentiation was detected among populations with a strikingly high value of $F_{\mathrm{ST}}$ (0.74) given the small geographical scale. This is even higher than the value reported by Charbonnel et al. (2002b) at similar geographic scale (0.57), and consistent with values reported in other selfing snail species (for example, Viard et al., 1997; Trouvé et al., 2003). However, populations were not isolated by distance.

Limited variability and strong population differentiation are the typical patterns in selfing species (Jarne, 1995; Charlesworth, 2003). This is due to lower effective population size, lower effective migration rate (when migration occurs at the diploid stage) and enhanced efficiency of purifying selection (the background selection effect; see Jarne, 1995; Charlesworth, 2003). Populations of freshwater snails are also experiencing cycles of drought and flood, which amplitude depends on habitat characteristics. Environmental stochasticity can result in repeated bottlenecks and extinctions (Städler and Jarne, 1997; Keller and Douglas, 2008). This was approached here through habitat openness (open versus closed) and permanence (temporary versus permanent). Indeed, genetic drift and extinction should be more common in temporary than in permanent habitats, and migration and colonization might maintain larger effective population size in open than in closed habitats (Bousset et al., 2004; Escobar et al., 2008). More variation is therefore expected in open than in closed, and in permanent than in temporary habitats. We failed though to detect any effect on genetic diversity (results not shown) contrary to previous work in freshwater snails (Bousset et al., 2004; Chapuis et al., 2007; Escobar et al., 2008).

A last explanation to the limited variability is that B. pfeifferi is a newcomer to the Man area. Indeed, urogenital schistosomiasis (due to S. haematobium) was the only form of schistosomiasis in this area up to the 1950s (Doumenge et al., 1987). This parasite is transmitted by both Bulinus truncatus and B. globosus in Côte d'Ivoire. Intestinal schistosomiasis, which is due to $S$. mansoni (transmitted by 


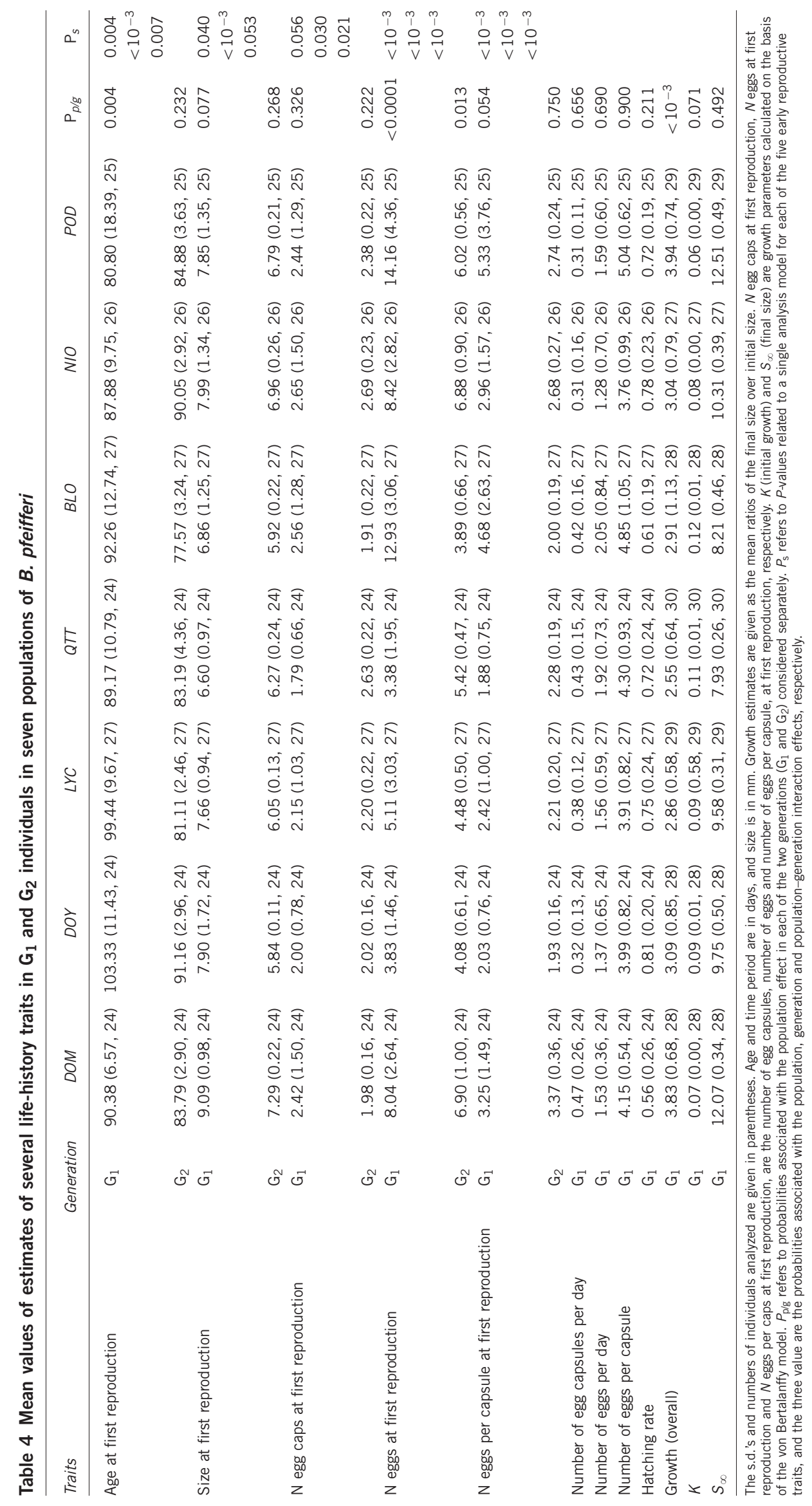


Table 5 Broad-sense heritability $\left(\mathrm{H}^{2} \pm\right.$ s.e.) of five traits characterizing early reproduction in seven populations of $B$. pfeifferi

\begin{tabular}{|c|c|c|c|c|c|c|c|}
\hline & $D O M$ & DOY & $L Y C$ & QTT & $B L O$ & $\mathrm{NIO}$ & $P O D$ \\
\hline Size & $0.44 \pm 0.19$ * & $0.50 \pm 0.18^{*}$ & $0.48 \pm 0.18^{*}$ & $0.15 \pm 0.21$ & $0.48 \pm 0.18^{*}$ & $0.26 \pm 0.20$ & $0.43 \pm 0.19$ * \\
\hline$N$ eggs & $0.38 \pm 0.20$ & $-0.04 \pm 0.21$ & $0.23 \pm 0.20$ & $0.34 \pm 0.20$ & $0.43 \pm 0.18^{*}$ & $0.36 \pm 0.19$ & $0.20 \pm 0.20$ \\
\hline$N$ eggs per capsule & $0.20 \pm 0.21$ & $-0.07 \pm 0.21$ & $0.17 \pm 0.20$ & $-0.02 \pm 0.21$ & $0.52 \pm 0.17^{* *}$ & $0.37 \pm 0.19$ & $-0.05 \pm 0.21$ \\
\hline
\end{tabular}

Boldface values were significantly different from zero. ${ }^{*} P<0.05,{ }^{* *} P<0.01,{ }^{* * *} P<0.001 . N$ egg capsules, $N$ eggs and $N$ eggs per capsule are the number of egg capsules, number of eggs and number of eggs per capsule, at first reproduction, respectively.

Table 6 Variance components and differentiation for quantitative traits, and neutral differentiation

\begin{tabular}{|c|c|c|c|c|c|c|c|c|}
\hline Traits & $\sigma^{2} H a b R e g$ & $\sigma^{2} \mathrm{HabOpn}$ & $\sigma^{2} P o p$ & $\sigma^{2}$ Fam & $\sigma^{2}$ Ind & $Q_{\text {HabRegTotal }}$ & $Q_{\text {HabOpnTotal }}$ & $Q_{P o p H a b}$ \\
\hline Age at first reproduction & 1536 & 864 & 274.22 & 509.43 & 133.52 & 0.66 & 0.52 & 0.35 \\
\hline Size at first reproduction & 2.41 & 3.15 & 2.37 & 2.6 & 1.33 & 0.33 & 0.39 & 0.48 \\
\hline$N$ egg caps at first reproduction & 0.67 & 1.5 & 1.8 & 2.35 & 0.9 & 0.14 & 0.27 & 0.43 \\
\hline$N$ egg at first production & 10.67 & 13.5 & 61.38 & 43.21 & 27.23 & 0.09 & 0.11 & 0.59 \\
\hline$N$ eggs per caps at first production & 4.45 & 7.71 & 7.66 & 3.54 & 2.29 & 0.28 & 0.41 & 0.68 \\
\hline $\begin{array}{l}\text { Mean } Q_{\mathrm{ST}} \\
\quad \mathrm{Cl}(95 \%)\end{array}$ & - & - & - & - & - & $\begin{array}{c}0.38 \\
0.12-0.47\end{array}$ & $\begin{array}{c}0.37 \\
0.22-0.46\end{array}$ & $\begin{array}{c}0.52 \\
0.40-0.61\end{array}$ \\
\hline $\begin{array}{l}\text { Overall } \boldsymbol{F}_{\mathrm{ST}} \\
\qquad \mathrm{Cl}(95 \%)\end{array}$ & - & - & - & - & - & $\begin{array}{c}0.07 \\
0.02-0.15\end{array}$ & $\begin{array}{c}0.20 \\
0.03-0.37\end{array}$ & $\begin{array}{c}0.74 \\
0.65-0.80\end{array}$ \\
\hline
\end{tabular}

The variance components are between habitats according to habitat regime $\left(\sigma^{2}\right.$ HabReg $)$ and openness $\left(\sigma^{2}\right.$ Habopn $)$, among populations within habitats $\left(\sigma^{2}\right.$ Pop $)$, and among families within populations $\left(\sigma^{2}\right.$ Fam $)$. The quantitative differentiation between habitats according to habitat regime $\left(Q_{\text {HabRegTotal }}\right)$ and openness $\left(Q_{\text {HabOpnTotal }}\right)$, and among populations within habitats $\left(Q_{\mathrm{PopHab}}\right)$ is given for each trait and over all traits. Confidence intervals (Cl) were obtained by bootstrapping traits. Corresponding values for $F_{S T}$ are also reported. Age at first Rep, Size at first Rep, $N$ egg caps at first reproduction, $N$ eggs at first reproduction and $N$ eggs per caps at first reproduction, are age, size and the number of egg capsules, number of eggs and number of eggs per capsule, at first reproduction, respectively. Mean $Q_{S T}$ is the mean phenotypic differentiation over the five life-history traits considered. Mean $F_{S T}$ is the mean genetic differentiation over the five microsatellite loci analysed.

B. pfeifferi), is today the only form of the disease in the Man area (Raso et al., 2005). B. pfeifferi has probably been introduced recently, as it has been in other areas (for example, Madagascar; Charbonnel et al., 2002b). Invasion is a further source of bottlenecks, and therefore of lower variability within populations (Wares et al., 2005; Keller and Douglas, 2008), though not of marked differentiation at such a geographic scale. On the other hand, no isolation by distance is expected if the area has been invaded recently because the migrationdrift equilibrium has not been reached.

\section{Life-history traits variation and population phenotypic differentiation}

Significant variation among the seven populations was detected for a subset only of the traits studied. This includes most traits related to early reproduction measured in both generations (for example, age and size at first reproduction), and overall growth in $G_{1}$ individuals. Previous studies have already shown marked variation in age and size at first reproduction, and in early fecundity among populations of freshwater snails, whether outcrossers (for example, Radix balthica: Evanno et al., 2006) or selfers (for example, G. truncatula: Chapuis et al., 2007). These are indeed key traits of animal life cycles (Roff, 1992; Stearns, 1992), and evidence is accumulating that this is also true in freshwater snails. Interestingly, positive correlations between trait pairs were detected in both $G_{1}$ and $G_{2}$ individuals with larger size/age at first reproduction associated with larger values of fecundity features. These correlations are consistent with previous results in freshwater snails, for example P. acuta (Tsitrone et al., 2003). This might be interpreted as resulting from selection on resource allocation. It is more surprising that significant correlations were not detected between overall growth (or its constituent parameters), and fecundity in $G_{1}$ individuals (note that no variation was observed in survival, such that we do not expect a correlation on that side) as expected from theoretical considerations on the evolution of lifehistory traits (for example, Roff, 2000).

An interesting result is that mean values of the five traits measured in both $G_{1}$ and $G_{2}$ individuals were larger in $G_{1}$ parents than in $G_{2}$ snails. No such trend was detected for the variances. This can be explained in several ways. The first explanation is a change in environmental conditions (for example, temperature, food) across generations, but we tried to maintain these conditions as constant as possible over the course of the whole experiment. A second explanation is the influence of maternal effects (see for example, Lynch and Walsh, 1998). They would have to be here directional and rather similar across populations. However, given the contrast in traits across habitats (see below) and genetic differentiation, we do not expect similar maternal effects across populations. This difference across generations did not prevent us though to detect significant heritabilities, perhaps because variance components were not affected.

A classical question is whether phenotypic variation is indicative of genetic variation in the traits studied. Early fecundity parameters are under strong stabilizing selection in many species (see for example, Roff, 2002), showing low heritability, and this is true in outcrossing individuals of the outcrossing freshwater snail P. acuta (Tsitrone et al., 2003; Escobar et al., 2008). In our study, significant heritability values were recorded for age and size at first reproduction in most populations (mean estimates: $0.43-0.67$ and $0.43-0.50$, respectively), though not for early fecundity parameters. These values might be overestimated though, as estimating early fecundity over a week only is associated with too much inter-individual variation. Chapuis et al. (2007), also studying a highly selfing snail, obtained much lower heritability values for age and size at first reproduction (0.24 and 0.11, respectively) perhaps as a result of selection, or due to dominance 
interactions or variation in experimental conditions (see for example, Falconer and Mackay, 1996). Similar explanations might hold for the non-significant heritability estimates observed for other traits in most populations in the present work.

A remarkable point is that significant heritability values were detected for two traits despite low genetic variability at neutral loci (see also Charbonnel et al., 2002a,b,c). A similar result was reported by Chapuis et al. (2007), as well as no correlation between gene diversity and genetic variance of traits (this is true here too; results not shown). Note that a correlation between neutral and selected diversity is not expected in general (reviewed in Leinonen et al., 2008). A first explanation is that our neutral loci are not representative of the true variability in B. pfeifferi populations. This is unlikely as the poor variation is typical of selfing species. A more plausible reason is the disruptive selection on these two key traits as a consequence of environmental variation. We showed (see discussion below) that traits are affected by habitat characteristics. Some temporal variation in environmental features, possibly associated with modest migration among populations experiencing different environmental conditions, may therefore explain the maintenance of significant quantitative variation within populations (Young and Clarke, 2000).

Indeed, populations occupying different environments may evolve different life-history strategies. Theoretical models predicts that organisms living in fluctuating environment should reproduce earlier, at a smaller size and produce larger clutch size compared to those living in stable habitats (review in Reznick et al., 2002). In freshwater habitats, water availability is a striking characteristic: permanent habitat could be classified as stable and temporary habitats as variable with regard to the probability of drying out (for example, Chapuis et al., 2007). Contrary to the above-mentioned prediction, snails from permanent habitats matured here at a smaller size/age and laid more eggs at first reproduction. A possible explanation is genuine selection for resistance to drought. Juvenile snails are known for their sensitivity to humidity stress (for example, Facon et al., 2004). Selection might therefore delay the onset of reproduction to a less fragile stage. Other selective factors, including parasites or predators, might have been involved as well. For example, the permanent site POD is used for fish breeding, and fish predation may accelerate reproduction onset. Habitat openness (closed versus open habitats) has also been shown to affect life-history traits (Escobar et al., 2008). We found here that reproduction was initiated later and at larger size in open than in closed habitats. The amount of available resources might be an explanation, but the other selective factors mentioned above should be kept in mind.

Spatial differentiation in molecular markers and life-history traits We obtained a $Q_{\mathrm{ST}}$ estimate over all populations of 0.52 (95\% CI: $0.40-0.61$ ), higher than the value reported in the single comparable study for almost the same traits $(0.28,95 \%$ CI: $0.19-0.34)$ in the selfing species G. truncatula (Chapuis et al., 2007). Moreover, we found $Q_{\mathrm{ST}}<F_{\mathrm{ST}}$ at the scale of all populations. The classical explanation is that stabilizing selection may have driven populations towards homogeneous phenotypic patterns. This explanation is plausible as the populations studied here are separated by a few kilometers, and are therefore probably under similar selection pressures. We also considered the incidence of habitat regime and openness. $Q_{\mathrm{ST}}$ values were not statistically different from $F_{\mathrm{ST}}$ values, suggesting that phenotypic divergence between open and closed habitats can be explained by drift alone (Merilä and Crnokrak, 2001; Chapuis et al., 2007). However, the Escobar et al. (2008) found
$F_{\text {st }}>Q_{\text {st }}$ between closed and open habitats of the outcrossing species $P$. acuta shows that habitat openness might affect phenotypic traits.

We are aware that our results should be considered with caution, especially because various processes can influence the $Q_{\mathrm{ST}}-F_{\mathrm{ST}}$ relationship. This includes selfing, which affects both dominance effects within loci and epistatic interactions among loci, maternal effects, and their variation across laboratory generations (to be considered here because we studied early expressed traits) and uncontrolled environmental variation (see Goudet and Büchi, 2006; Chapuis et al., 2007 for review). On the whole though, they tend to depress, rather than inflate, $Q_{\mathrm{ST}}$. Another issue is that the comparison between $Q_{\mathrm{ST}}$ and $F_{\mathrm{ST}}$ remains valid provided that the underlying mutation rates are comparable (Edelaar and Björklund, 2011). This casts some doubts on studies estimating neutral variability using microsatellite data, because their high variability may strongly depress $F_{\mathrm{ST}}$ estimates. It is unlikely that this holds $\left(F_{\mathrm{ST}}\right.$ estimates are whatsoever pretty high) because, even in highly selfing species, highly variable microsatellites would produce much higher within-population variability than observed here (compare with for example, Viard et al., 1997). Both Whitlock and Guillaume (2009) and Ovaskainen et al. (2011) raised statistical issues when comparing $Q_{\mathrm{ST}}$ and $F_{\mathrm{ST}}$. The method of Whitlock and Guillaume (2009) is difficult to implement as typical data sets (as ours) rarely include enough loci to directly infer the distribution of $F_{\mathrm{ST}}$ without extra inferential steps, and $Q_{\mathrm{ST}}$ for a trait is rarely measured with high precision, so the position of a given estimated $Q_{\mathrm{ST}}$ value in the distribution cannot be known without error. The approach promoted by Ovaskainen et al. (2011) seems to be the most appropriate at this point, but is unfortunately too intricate to be conducted in a convenient way.

As a conclusion, selection among habitats characterized by different water availability is therefore a serious candidate for driving phenotypic and genetic differentiation of traits, and should be considered in studies of traits in freshwater snails. We are pleading for further work based on a larger number of populations, or using an experimental approach under controlled drought conditions in the laboratory or using mesocosms.

\section{DATA ARCHIVING}

Data deposited in the Dryad repository: doi:10.5061/dryad.pq56h.

\section{CONFLICT OF INTEREST}

The authors declare no conflict of interest.

\section{ACKNOWLEDGEMENTS}

This work received financial support from the Swiss Agency for Development and Cooperation (SDC) allocated to the Center Suisse de Recherches Scientifiques en Côte d'Ivoire (CSRS) via a project entitled 'Contribution to the process of national reconciliation in Côte d'Ivoire' (AVS). We gratefully acknowledge the Swiss Tropical and Public Health Institute (Swiss TPH) for having entirely supported the purchase of all the genetic analysis materials. We are also grateful to the Center National de Recherche Agronomique (CNRA) for having allowed snail genotyping in the Laboratoire Central de Biotechnologie (LCB).

Bell G (2008). Selection: the Mechanism of Evolution. Oxford University Press.

Bousset L, Henry P-Y, Sourrouille P, Jarne P (2004). Population biology of the invasive freshwater snail Physa acuta approached through genetic markers, ecological characterization and demography. $\mathrm{Mol}$ Ecol 13: 2023-2036.

Brown D (1994). Freshwater Snails of Africa and Their Medical Importance, 2nd edn. Taylor and Francis Ltd: London. 
Chapuis E, Trouve S, Facon B, Degen L, Goudet J (2007). High quantitative and no molecular differentiation of a freshwater snail (Galba truncatula) between temporary and permanent water habitats. Mol Ecol 16: 3484-3496.

Charbonnel N, Angers B, Razatavonjizay R, Bremond P, Jarne P (2000). Microsatellite variation in the freshwater snail Biomphalaria pfeifferi. Mol Ecol 9: 993-1011.

Charbonnel N, Angers B, Rasatavonjizay R, Bremond P, Jarne P (2002a). Evolutionary aspects of the metapopulation dynamics of Biomphalaria pfeifferi, the intermediate host of Schistosoma mansoni. J Evol Biol 15: 248-261.

Charbonnel N, Angers B, Rasatavonjizay R, Bremond P, Debain C, Jarne P (2002b). The influence of mating system, demography, parasites and colonisation on the population structure of Biomphalaria pfeifferi in Madgascar. Mol Ecol 11: 2213-2228.

Charbonnel N, Quesnoit M, Razatavonjizay R, Brémond P, Jarne P (2002c). A spatial and temporal approach to microevolutionary forces affecting population biology in the freshwater snail Biomphalaria pfeifferi. Am Nat 160: 741-755.

Charbonnel N, Rasatavonjizay R, Sellin E, Brémond P, Jarne P (2005). The influence of genetic factors and population dynamics on the mating system of the hermaphroditic snail Biomphalaria pfeifferi. Oikos 108: 283-296.

Charlesworth D (2003). Effects of inbreeding on the genetic diversity of populations. Philos Trans R Soc Lond Biol Sci 358: 1051-1070.

Crawley MJ (2005). Statistics - An Introduction Using R. Wiley.

David P, Pujol B, Viard F, Castella V, Goudet J (2007). Reliable selfing rate estimates from imperfect population genetic data. Mol Ecol 16: 2474-2487.

Dillon RT (2000). The Ecology of Freshwater Molluscs. University Press: Cambridge.

Doumenge J-P, Mott KE, Cheung C, Villenave D, Chapuis C, Perrin MF et al. (1987). Atlas of the Global Distribution of Schistosomiasis. Centre d'Etudes de Géographie Tropicale (C.N.R.S.)/World Health Organization, Presse Universitaires de Bordeaux: Talence.

Edelaar PIM, Björklund M (2011). If $F_{\text {ST }}$ does not measure neutral genetic differentiation, then comparing it with $Q_{S T}$ is misleading. Or is it? Mol Ecol 20: 1805-1812.

Edelaar P, Burraco P, Gomez-Mestre I (2011). Comparisons between Qst and Fst - how wrong have we been? Mol Ecol 20: 4830-4839.

Escobar JS, Nicot A, David P (2008). The difference sources of variation in inbreeding depression, heterosis and outbreeding depression in a metapopulation of Physa acuta. Genetics 180: 1593-1608.

Escobar JS, Auld JR, Correa AC, Alonso JM, Bony YK, Koene JM et al. (2011). Patterns of mating-system evolution in hermaphroditic animals: correlations among selfing rate, inbreeding depression and delayed selfing: patterns of mating-system evolution in hermaphroditic animals. Evolution 65: 1233-1253.

Evanno G, Castella E, Goudet J (2006). Evolutionary aspects of population structure for molecular and quantitative traits in the freshwater snail Radix balthica. J Evol Biol 19: 1017-1082.

Facon B, Machline E, Pointier JP, David P (2004). Variation in desiccation tolerance in freshwater snails and its consequences for invasion ability. Biol Invasions 6: 283-293.

Falconer DS, Mackay TFC (1996). Introduction to Quantitative Genetics. Longman: New York.

Goudet J, Büchi L (2006). The effects of dominance, regular inbreeding and sampling design on $Q_{s t}$, an estimator of population differentiation for quantitative traits. Genetics 172: $1337-1347$

Hartl DL, Clark AG (1997). Principles of Population Genetics, 3rd edn. Sinauer Associates: Sunderland, MA.

Holsinger KE, Weir BS (2009). Genetics in geographically structured population: defining, estimating and interpreting $F_{\mathrm{ST}}$. Nat Rev Genet 10: 639-650.

Jarne $P$ (1995). Mating system, bottlenecks and genetic polymorphism in hermaphroditic animals. Genet Res 65: 193-207.
Jarne P, Delay B (1991). Population genetics of freshwater snails. Trends Ecol Evol 6 : 383-386.

Keller SR, Douglas RT (2008). History, chance and adaptation during biological invasion: separating stochastic phenotypic evolution from response to selection. Ecol Lett 11: 852-866

Leinonen T, O'Hara R, Cano JM, Merilä J (2008). Comparative studies of quantitative trait and neutral marker divergence: a meta-analysis. J Evol Biol 21: 1-17.

Loreau M, Baluku B (1987). Growth and demography of populations of Biomphalaria pfeifferi (Gastropoda, Planorbidae) in the laboratory. J Molluscan Stud 53: 171-177.

Lynch M, Walsh B (1998). Genetics and Analysis of Quantitative Traits. Sinauer Associates: Sunderland, MA.

Merilä J, Crnokrak P (2001). Comparison of genetic differentiation at marker loci and quantitative traits. J Evol Biol 14: 892-903.

Nei M (1987). Molecular Evolutionary Genetics. Columbia University Press: New York.

Ovaskainen O, Karhunen M, Zheng C, Arias JMC, Merilä J (2011). A new method to uncover signatures of divergent and stabilizing selection in quantitative traits. Genetics 189: 621-632.

Pujol B, Wilson AJ, Rossi RIC, Pannell JR (2008). Are $Q_{S T}-F_{S T}$ comparisons for natural populations meaningful? Mol Ecol 17: 4782-4785.

Raso G, Matthys B, N'Goran EK, Tanner M, Vounatsou P, Utzinger J (2005). Spatial risk prediction and mapping of Schistosoma mansoni infections among schoolchildren living in western Côte d'Ivoire. Parasitology 131: 97-108.

Reznick D, Bryant MJ, Bashey F (2002). r- and K-selection revisited: the role of population regulation in life-history evolution. Ecology 83: 1509-1520.

Roff DA (1992). The Evolution of Life Histories. Theory and Analysis. Chapman \& Hall: New York.

Roff DA (1997). Evolutionary Quantitative Genetics. Chapman \& Hall: New York.

Roff DA (2000). Trade-offs between growth and reproduction: an analysis of the quantitative genetic evidence. J Evol Biol 13: 434-445.

Roff DA (2002). Life History Evolution. Sinauer Associates: Sunderland, MA.

Rousset F (2007). Genepop 4.0 for Windows and Linux.

Städler T, Jarne P (1997). Population biology, genetic structure, and mating system parameters. Streit B, Städler T, Lively CM (eds). Ecology and Evolution of Freshwater Organisms. Birkhäuser Verlag: Basel. pp 231-262.

Stearns SC (1992). The Evolution of Life Histories. Oxford University Press: New York.

Tian-Bi TY-N, N'Goran KE, N'Guetta S-P, Matthys B, Sangaré A, Jarne P (2008). Prior selfing and the selfing syndrome in animals: an expiremental approach in the freshwater snail Biomphalaria pfeifferi. Genet Res 90: 61-72.

Trouvé S, Degen L, Renaud F, Goudet J (2003). Evolutionary implications of a high selfing rate in the freshwater snail Lymnaea truncatula. Evolution 57: 2303-2314.

Tsitrone A, Jarne P, David P (2003). Delayed selfing and resource reallocation in relation to mate availability in the freshwater snail Physa acuta. Am Nat 162: 474-488.

Viard F, Justy F, Jarne P (1997). Population dynamics inferred from temporal variation at microsatellite loci in the selfing snail Bulinus truncatus. Genetics 146: 973-982.

Wares JP, Hughes AR, Grosberg RK (2005). Mechanisms that drive evolutionary change: insights from species introductions and invasions. Sax DF, Stachowicz JJ, Gaines SD (eds). Species Invasions: Insights into Ecology, Evolution, and Biogeography. Sinauer Associates: Sunderland, MA. pp 229-257.

Weir BS, Cockerham CC (1984). Estimating F-statistics for the analysis of population structure. Evolution 38: 1358-1370.

Whitlock MC, Guillaume F (2009). Testing for spatially divergent selection: comparing $Q_{S T}$ to $F_{\mathrm{ST}}$. Genetics 183: 1055-1063.

Young AG, Clarke GM (2000). Genetics, Demography and Viability of Fragmented Populations. Cambridge University Press: Cambridge.

Supplementary Information accompanies the paper on Heredity website (http://www.nature.com/hdy) 